\title{
What a speaker's choice of frame reveals: Reference points, frame selection, and framing effects
}

\author{
CRAIG R. M. MCKENZIE and JONATHAN D. NELSON \\ University of California, San Diego, La Jolla, California
}

\begin{abstract}
Framing effects are well established: Listeners' preferences depend on how outcomes are described to them, or framed. Less well understood is what determines how speakers choose frames. Two experiments revealed that reference points systematicallyinfluenced speakers' choices between logically equivalent frames. For example, speakers tended to describe a 4-ounce cup filled to the 2-ounce line as half full if it was previously empty but described it as half empty if it was previously full. Similar results were found when speakers could describe the outcome of a medical treatment in terms of either mortality or survival (e.g., 25\% die vs. 75\% survive). Two additional experiments showed that listeners made accurate inferences about speakers' reference points on the basis of the selected frame (e.g., if a speaker described a cup as half empty, listeners inferred that the cup used to be full). Taken together, the data suggest that frames reliably convey implicit information in addition to their explicit content, which helps explain why framing effects are so robust.
\end{abstract}

Framing effects refer to the fact that logically equivalent rewordings of outcomes or attributes can affect people's preferences or judgments. Tversky and Kahneman's (1981) "Asian disease problem" illustrates this phenomenon in the context of risky choice:

Imagine that the U.S. is preparing for the outbreak of an unusual Asian disease, which is expected to kill 600 people. Two alternative programs to combat the disease have been proposed. Assume that the exact scientific estimate of the consequences of the programs are as follows:

If Program A is adopted, 200 people will be saved.

If Program B is adopted, there is $1 / 3$ probability that 600 people will be saved, and $2 / 3$ probability that no people will be saved.

A majority of the participants selected Program A over B. A separate group was presented with the same cover story, but with the two programs reformulated:

If Program C is adopted, 400 people will die.

If Program $D$ is adopted, there is $1 / 3$ probability that nobody will die, and $2 / 3$ probability that 600 people will die.

Although Program C is simply a rewording of A and Program $\mathrm{D}$ is a rewording of $\mathrm{B}$, only a minority of the par-

This research was supported by National Science Foundation Grant SES-0079615. Some of the results were presented at the 2001 Annual Meeting of the Psychonomic Society, Orlando, FL. The authors thank Vic Ferreira, Gideon Keren, Irwin Levin, Shlomi Sher, X. T. Wang, and Mike Ziolkowski for thoughtful comments on earlier drafts of this manuscript. Correspondence should be addressed to C. R. M. McKenzie, Department of Psychology, University of California, San Diego, La Jolla, CA 92093-0109 (e-mail: cmckenzie@ucsd.edu), or to J. D. Nelson, UCSD Cognitive Science Department 0515, La Jolla, CA 920930515 (jnelson@ cogsci.ucsd.edu). ticipants preferred $\mathrm{C}$ to $\mathrm{D}$. That is, group preferences reversed when the options were merely rephrased. Programs A and B are phrased in terms of lives saved, with the implicit reference point that 600 lives will be lost. People are generally risk averse for gains and prefer the certain option, or A. Programs C and D are phrased in terms of lives lost, with the implicit reference point that no lives will be lost. Because people are generally risk seeking for losses, participants prefer the uncertain option, or D (Kahneman \& Tversky, 1979; see also Kahneman \& Tversky, 1984; Tversky \& Kahneman, 1981, 1986). Framing effects on risky choice have been demonstrated with a wide variety of tasks (for reviews, see Kühberger, 1998; Levin, Schneider, \& Gaeth, 1998).

Framing effects also occur with simpler tasks. For example, describing a medical treatment outcome in terms of $X \%$ survival rather than $100-X \%$ mortality increases its perceived acceptability (Levin, Schnittjer, \& Thee, 1988; Marteau, 1989; McNeil, Pauker, Sox, \& Tversky, 1982; Wilson, Kaplan, \& Schneiderman, 1987; see also Meyerowitz \& Chaiken, 1987). Similarly, Levin and Gaeth (1988) found that describing ground beef as $75 \%$ lean rather than as $25 \%$ fat led participants to rate it as being leaner, of higher quality, and less greasy (see also Moxey \& Sanford, 2000; Sanford, Fay, Stewart, \& Moxey, 2002). As with risky choice, framing effects due simply to the relabeling of attributes have been widely demonstrated (Levin et al., 1998).

As the above studies illustrate, research on framing effects has shown that listeners, or those presented with frames, behave differently depending on the frame. The focus of this article, by contrast, is on what determines how speakers spontaneously frame outcomes. Given that frames have a powerful influence on listeners' preferences, what deter- 
mines which frame a speaker selects? For instance, what affects whether the "scientific estimate" in the Asian disease problem would be phrased in terms of lives saved or lives lost, and what affects whether someone describes ground beef in terms of how fat or lean it is?

We propose that the frame (e.g., percentage who die vs. percentage who survive, percentage of fat vs. percentage of lean) chosen by speakers to describe a current situation tends to be the one that has increased relative to their reference point. For example, the programs to combat the Asian disease might be more likely to be framed in terms of lives lost if no one had ever died from the Asian disease before (and hence, zero deaths was the reference point) than if the disease had routinely killed 600 people each year. Similarly, perhaps ground beef would be more likely to be described in terms of $25 \%$ fat if most ground beef had less, rather than more, fat.

This view of how reference points affect frame selection is illustrated in Figure 1. For present purposes, the domain of interest involves a dimension (e.g., percentage of people who die), any point on which can be described by two labels with opposite meanings (e.g., die vs. survive). In such cases, any given state can be phrased in one of two logically equivalent ways: Either X\% Label 1 or $100-X \%$ Label 2 . The key idea is that, given a current situation, the label (and hence, the frame) selected by a speaker will be influenced by the speaker's reference point. Specifically, for a given current situation, a particular label is more likely to be used when that label's percentage has increased, rather than decreased, relative to the reference point. In Figure 1, the current situation is more likely to be described in terms of Label 1 if the reference point is A rather than B. Equivalently, the same situation is more likely to be described in terms of Label 2 if the reference point is $B$ rather than $A$. These qualitative predictions should hold true even if (as we suspect will often be the case) there is a general tendency to prefer one label to the other.

Our account of frame selection is based largely on intuition, but it is worth noting that (English) speakers appear to have a general tendency to use terms that correspond to the label (or pole) that has increased. For example, a person whose height has increased is usually referred to as taller, not less short, whereas a person whose height has decreased (i.e., shortness has increased) is usually referred to as shorter, not less tall. Note further that there is no morpheme in English that is analogous to the suffix -er to indicate that a dimension has decreased, which also seems to imply that increasing labels or poles have a special status.

The important implication of this analysis is that, if reference points reliably influence speakers' frame selection, then frames carry information beyond their literal content. Frames that are logically equivalent might, nonetheless, convey different information. Specifically, the selected frame would provide evidence of the speaker's reference point. (For a general discussion of the role of listeners' conversational assumptions in interpreting judgment tasks, see Hilton, 1995, and Schwarz, 1996; for evidence that listeners sometimes feel that logically equivalent frames should be treated differently, albeit for different reasons from those discussed here, see Frisch, 1993.)

Assuming that frame selection is influenced by reference points and that, therefore, frames carry surplus information, a natural question is whether listeners reliably pick up that information. For instance, if a speaker chose to frame the Asian disease programs in terms of lives lost, would a listener infer that few people usually die from the disease? In fact, a key aspect of the usual explanation of risky choice framing effects is that presented frames influence listeners' reference points (e.g., Schneider, 1992; Tversky \& Kahneman, 1981). A novel contribution of our research, beyond examining frame selection, was to examine whether listeners' inferences about reference points would correspond to the actual reference points that influenced the speaker's choice of frame. We tested the following three hypotheses: (1) Speakers' frame selection is reliably influenced by reference points, (2) listeners make inferences about speakers' reference points on the basis of presented frames, and (3) the reference points in (1) and (2) - actual and inferred, respectively-match. We are aware of no previous research that has examined these issues together. If these three hypotheses were to be confirmed, it would indicate that frame selection serves an efficient communicative function by reliably conveying implicit information in addition to the explicit informa-

\begin{tabular}{|c|c|c|c|c|}
\hline & $\begin{array}{c}\text { Reference } \\
\text { Point A }\end{array}$ & $\begin{array}{l}\text { Current } \\
\text { Situation }\end{array}$ & $\begin{array}{c}\text { Reference } \\
\text { Point B }\end{array}$ & \\
\hline Label 1: $\quad 0 \%$ & $1-$ & $\rightarrow$ & 1 & $100 \%$ \\
\hline Label 2: $100 \%$ & $\begin{array}{l}3 \\
1\end{array}$ & $\leftarrow$ & -1 & $0 \%$ \\
\hline
\end{tabular}

Figure 1. Illustration of how reference points a re hypothesized to influence frame selection. Generally, it is predicted that a particular label is more likely to be used to describe a given current situation when that label's percentage has increased (rather than decreased) relative to a reference point. For example, imagine that the current status of a cup is that it is one-half full (one-half empty), and let Label 1 be "full," Label 2 be "empty," Reference Point A be one-quarter full (threequarters empty), and Reference Point $B$ be three-quarters full (one-quarter empty ). It is predicted that participants will be more likely to describe the cup as one-half full when the reference point is one-quarter full rather than three-quarters full. Equivalently, it is predicted that participants will be more likely to describe the cup as one-half empty when the reference point is three-quarters full rather than one-quarter full. 
tion. This additional communicative aspect of frames might, in turn, help explain why logically equivalent frames lead to different behavior on the part of listeners.

In our first two experiments, we examined whether speakers' frame selection depends on their reference point. ${ }^{1}$ In our last two experiments, we examined whether listeners pick up any surplus information that frames might contain regarding speakers' reference points.

\section{EXPERIMENT 1}

\section{Method}

The participants were 192 University of California, San Diego (UCSD) students, who received partial course credit. There were 32 participants in each of six conditions. In one condition, the participants read the following half-full-half-empty scenario:

Imagine a 4-ounce measuring cup in front of you that is completely filled with water up to the 4-ounce line. You then leave the room briefly and come back to find that the water is now at the 2-ounce line. What is the most natural way to describe the cup now?

They selected either "The cup is $1 / 2$ full" or "The cup is $1 / 2$ empty." (The order of options was reversed for half of the participants in each condition in each experiment reported here.) The participants in the second condition read the scenario, but the cup was originally empty rather than full. Thus, in both of these half-full- half-empty scenarios, the cup to be described was the same-namely, filled to the 2ounce line. The difference between the scenarios was the state of the cup before the change (i.e., the reference point). At issue was whether the reference point would influence which of the two logically equivalent descriptions was preferred.

Other participants were presented with a one-quarter-full-threequarters-empty scenario. As in the above scenario, the cup started out full for some participants (third condition) and started out empty for others (fourth condition). The final state of the cup was the same for both conditions: Water was at the 1-ounce line. The participants selected the statement they felt best described the cup at that point: either "The cup is $1 / 4$ full" or "The cup is $3 / 4$ empty."

In the three-quarters-full- one-quarter-empty scenario, the cup started out either full or empty (fifth and sixth conditions), and the final state was water at the 3-ounce line. The options were "The cup is $3 / 4$ full" and "The cup is $1 / 4$ empty."

\section{Results and Discussion}

The results are shown in the top of Table 1. For the halffull-half-empty scenario, only $31 \%$ of the participants chose to describe the 4-ounce cup with water at the 2ounce line as "half full" when it had previously been full (i.e., most preferred to describe it as half empty), but $88 \%$ described the same cup as "half full" when it had previ-

Table 1

Percentage of Responses in Each Condition for Experiments 1-4

\begin{tabular}{|c|c|}
\hline \multicolumn{2}{|c|}{ Experiment 1 (Glass Speaker Study) } \\
\hline Half-full-half-empty scenario & $\%$ selecting "half-full" frame \\
\hline 4 ounces $\rightarrow 2$ ounces & 31 \\
\hline 0 ounces $\rightarrow 2$ ounces & 88 \\
\hline One-quarter full-three-quarters-empty & $\%$ selecting "one-quarter full" frame \\
\hline 4 ounces $\rightarrow 1$ ounce & 56 \\
\hline 0 ounces $\rightarrow 1$ ounce & 97 \\
\hline Three-quarters-full-one-quarter-empty & $\%$ selecting "three-quarters full" frame \\
\hline 4 ounces $\rightarrow 3$ ounces & 75 \\
\hline 0 ounces $\rightarrow 3$ ounces & 88 \\
\hline \multicolumn{2}{|c|}{ Experiment 2 (Medical Speaker Study) } \\
\hline $50 \%$ survive $-50 \%$ die & $\%$ selecting " $50 \%$ survive" frame \\
\hline $100 \%$ survive $\rightarrow 50 \%$ survive & 41 \\
\hline $0 \%$ survive $\rightarrow 50 \%$ survive & 94 \\
\hline $25 \%$ survive $-75 \%$ die & $\%$ selecting " $25 \%$ survive" frame \\
\hline $50 \%$ survive $\rightarrow 25 \%$ survive & 50 \\
\hline $0 \%$ survive $\rightarrow 25 \%$ survive & 84 \\
\hline $75 \%$ survive $-25 \%$ die & $\%$ selecting " $75 \%$ survive" frame \\
\hline $100 \%$ survive $\rightarrow 75 \%$ survive & 53 \\
\hline $50 \%$ survive $\rightarrow 75 \%$ survive & 80 \\
\hline \multicolumn{2}{|c|}{ Experiment 3 (Glass Listener Study) } \\
\hline Half full-half empty & $\%$ inferring "was full" reference point \\
\hline$? \rightarrow$ "half full" & 50 \\
\hline$? \rightarrow$ "half empty" & 80 \\
\hline \multicolumn{2}{|l|}{ One-quarter full-three-quarters empty } \\
\hline$? \rightarrow$ “one-quarter full" & 35 \\
\hline$? \rightarrow$ "three-quarters empty" & 94 \\
\hline \multicolumn{2}{|l|}{ Three-quarters-full-one-quarter-empty } \\
\hline$? \rightarrow$ "three-quarters full" & 56 \\
\hline$? \rightarrow$ "one-quarter empty" & 79 \\
\hline \multicolumn{2}{|c|}{ Experiment 4 (Medical Listener Study) } \\
\hline $50 \%$ survive $-50 \%$ die & $\%$ inferring " $100 \%$ survive" reference point \\
\hline$? \rightarrow$ "50\% survive" & 34 \\
\hline$? \rightarrow$ "50\% die" & 52 \\
\hline
\end{tabular}


ously been empty ( $p<.001 ; p$ values correspond to twotailed Fisher's exact tests, unless noted otherwise). Similarly, for the one-quarter-full-three-quarters-empty scenario, slightly more than half of the participants preferred to describe the cup with water at the 1-ounce line as "onequarter full" when the cup had been full, but virtually all did so when the cup had been empty $(p<.001)$. Finally, for the three-quarters-full-one-quarter-empty scenario, in which the cup had water at the 3-ounce line, the participants were more likely to describe the cup as "three-quarters full" when the cup had been empty rather than full, but the effect was not significant ( $p=.34$ ).

Taken together, these results show that the participants were not indifferent between logically equivalent descriptions of a cup's contents when there was a clear reference point. Consistent with the theoretical account illustrated in Figure 1, the participants were more likely to describe the cup in terms of how full it was if it was previously empty than if it was previously full.

Although the reference point influenced the selected frame, there was a general preference for describing the cup in terms of how full, rather than how empty, it was ( $72 \%$ across all conditions; $p<.001$, binomial test). This was especially true in the three-quarters-full-one-quarter-empty scenario, in which the participants were reluctant to describe a cup that was mostly full in terms of how empty it was, regardless of the reference point. Despite the general preference for describing cups in terms of how full they are, there were no qualitative violations of the predictions in any scenario.

\section{EXPERIMENT 2}

In our second experiment, we used a medical scenario in which a treatment's outcome could be described as either $X \%$ of patients survive or $100-X \%$ of patients die. As was mentioned, others have shown that rewording treatment outcomes affects preferences (Levin et al., 1988; Marteau, 1989; McNeil et al., 1982; Wilson et al., 1987). Experiment 2 tested the robustness of our earlier findings, using content with important practical relevance.

\section{Method}

The participants were 188 UCSD students, and there were between 30 and 32 participants in each of six conditions. In one condition, the participants read the following 50\%-survive-50\%-die scenario:

Imagine a disease that leads to many unpleasant symptoms and can even cause death. For the past 20 years, the same treatment has been used in patients with the disease. In terms of mortality, $100 \%$ of patients with this treatment die within 5 years; $0 \%$ survive. A new treatment has been tested, and it has several advantages and disadvantages. In terms of mortality, $50 \%$ of patients with the new treatment die within 5 years; $50 \%$ survive. Given that the usual treatment has been used for years, what is the most natural way to phrase the mortality results of the new treatment?

The participants selected either " $50 \%$ of patients survive" or " $50 \%$ of patients die." In a second condition, the new treatment's efficacy was the same, as were the options, but the old treatment's efficacy was different: $100 \%$ survive. (Note that the treatment outcomes in the scenario were in terms of both survival and mortality rates, but we will refer only to survival rates for the sake of exposition.) For the third and fourth conditions, $25 \%$ of patients survive with the new treatment, but the old treatment's efficacy was either $0 \%$ survival or $50 \%$ survival, respectively. The two options for describing the new treatment were " $25 \%$ of patients survive" and " $75 \%$ of patients die." For the two final conditions, $75 \%$ of patients survive with the new treatment, but the old treatment led either $50 \%$ or $100 \%$ of patients to survive. In either case, the two options for describing the new treatment were " $75 \%$ of patients survive" and " $25 \%$ of patients die." Note that in this experiment, we used intermediate reference points (50\% survival/mortality) in addition to the extreme ones $(0 \%$ and $100 \%$ ) in Experiment 1.

\section{Results and Discussion}

The results are shown in Table 1 . For the $50 \%$-survive$50 \%$-die scenario, only $41 \%$ of the participants chose to describe the new treatment results as " $50 \%$ of patients survive" when the old treatment led to $100 \%$ survival, but $94 \%$ did so when the old treatment led to $0 \%$ survival $(p<.001)$. For the $25 \%$-survive-75\%-die scenario, the participants were more likely to describe the new treatment results as " $25 \%$ of patients survive" when the old treatment led to $0 \%$ survival than when it led to $50 \%$ survival $(p=.007)$. Finally, for the $75 \%$-survive-25\%-die scenario, the participants were marginally significantly more likely to describe the new treatment results as " $75 \%$ of patients survive" when the old treatment led to $50 \%$ survival than when it led to $100 \%$ survival ( $p=.054)$.

These results replicate those of Experiment 1 and show that reference points have systematic effects on which of two logically equivalent frames is chosen. Analogous to the earlier results and in agreement with our theoretical account, the participants were more likely to describe the new treatment's results in terms of $X \%$ survive if it led to a higher, rather than lower, survival rate, relative to the old treatment. Furthermore, also as in Experiment 1, there was an overall preference for one of the labels: Across conditions, the participants preferred to describe the new treatment in terms of survival rather than mortality $(67 \% ; p<$ .001 , binomial test). Despite this overall preference for one label, there were again no qualitative violations of the predictions in any scenario. Thus, similar results were found with entirely different content and by using intermediate, in addition to extreme, reference points.

\section{EXPERIMENT 3}

The fact that speakers reliably select frames depending on their reference points means that there is surplus information contained in frames. For example, in Experiment 1, a cup described in terms of how empty it is was more likely to have been previously full rather than empty, and in Experiment 2, when a new medical treatment outcome was described in terms of how many people survive, it was more likely that the old treatment led to a higher, rather than a lower, mortality rate. Generally, listeners should be able to infer a speaker's reference point on the basis of which frame the speaker has chosen. Reference point information contained in frames might often be important to listeners. For instance, a physician's 
choice of frame might indicate to a patient whether a treatment has a relatively high or low survival rate. Do listeners accurately infer speakers' reference points from frames?

The predictions are the mirror image of those for frame selection, and Figure 1 again provides a guide: Listeners are expected to be more likely to infer that the speaker's reference point was A when the current situation is described by the speaker in terms of Label 1 rather than in terms of Label 2.

\section{Method}

The participants were 205 UCSD students, and there were 34 or 35 participants in each of six conditions. In one condition, they read the following half-full-half-empty scenario:

Imagine that Mary was sitting at her kitchen table with a glass in front of her. She left the room briefly and came back to find that the contents of the glass had changed. When asked to describe the glass now, Mary said, "The glass is half full." Given how Mary chose to describe the glass after its contents had changed, please choose the statement below in terms of what you think was most likely true about the glass before its contents changed.

The participants then selected either "The glass was full before its contents changed" or "The glass was empty before its contents changed." In a second condition, the participants read the same scenario, except that Mary described the glass as "half empty."

In the two one-quarter-full-three-quarters-empty conditions, the glass was described as either one-quarter full or three-quarters empty, and the participants responded whether the glass used to be full or empty. In the two three-quarters-full- one-quarter-empty conditions, the glass was described as either three-quarters full or onequarter empty, and the participants responded whether the glass used to be full or empty.

\section{Results and Discussion}

The results are shown in Table 1. When Mary described the glass as currently half full, $50 \%$ of the participants responded that the glass was more likely to have previously been full rather than empty, but when Mary described the glass as half empty, $80 \%$ reported that it was more likely that the glass had been full $(p=.012)$. Similarly, when the glass was described as one-quarter full, few participants thought it likely that it was previously full, but when it was described as three-quarters empty, most thought that it had been full $(p<.001)$. Finally, fewer participants thought the glass had previously been full when Mary described it as three-quarters full rather than one-quarter empty, but the effect was only marginally significant ( $p=.068$ ).

Thus, the listeners made different inferences about the speaker's reference point on the basis of the frame selected by the speaker, and they did so in a manner consistent with the predictions: They were more likely to infer that the glass was previously full if it was described by the speaker in terms of how empty, rather than how full, it was. Importantly, this implies that listeners make generally accurate inferences about speakers' reference points.

The designs used in Experiments 1 and 3 were essentially mirror images of each other. Experiment 1 provided speakers with a reference point and asked for a frame, and Experiment 3 provided listeners with a frame and ask them to infer the speaker's reference point. Plotted in Figure 2 are the results of the two experiments, paired by cor- responding conditions. On the $x$-axis is the proportion of participants in each scenario in Experiment 1 who were given the full reference point, conditional on whether they selected the empty frame or the full frame (or $p$ [container was full | speaker's frame]). On the $y$-axis is the proportion of participants in each scenario in Experiment 3 who inferred that the speaker's reference point was full, conditional on the frame presented to them (or $p$ [listener infers container was full $\mid$ speaker's frame]). For example, the data point in the upper right corner of the figure is due to the fact that (1) of the participants in Experiment 1 who chose the three-quarters empty frame, $94 \%$ of them had the full rather than the empty reference point $[.44 /(.44+$ $.03)=.94]$, and (2) of the participants in Experiment 3 who were presented with the three-quarters empty frame, $94 \%$ of them inferred that the glass was more likely previously full rather than empty. The fact that most of the data points lie close to the identity line shows that the listeners tended to infer a particular reference point, given a particular frame, about as often as that really was the speakers' reference point, given the speaker's chosen frame. This suggests that listeners might be surprisingly good at inferring speakers' reference points. ${ }^{2}$

To make sure that these listener results held in a different context, we conducted an additional experiment that

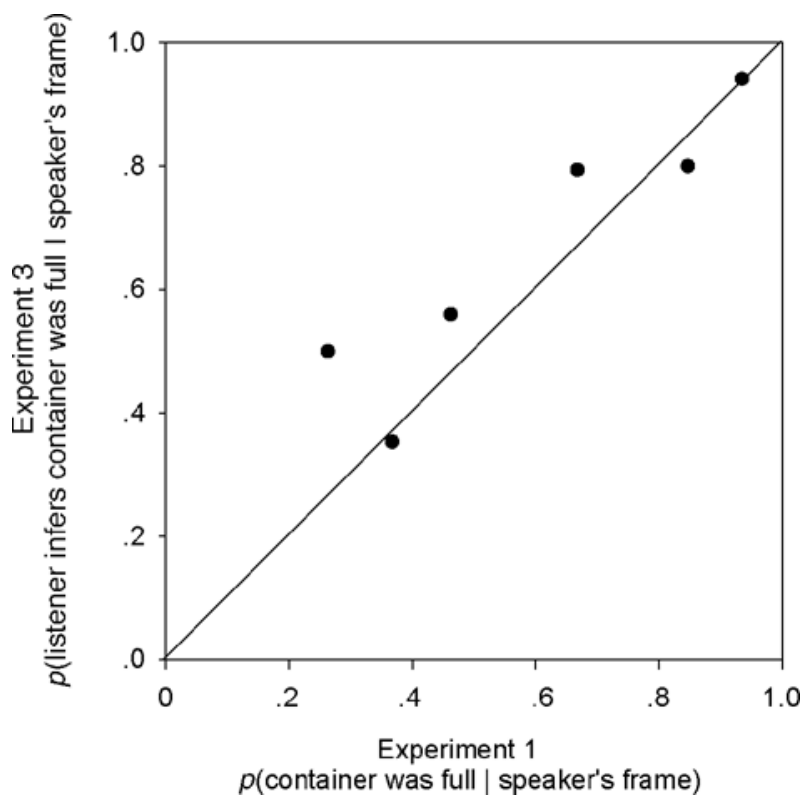

Figure 2. Plotted are the results of Experiments 1 and 3, paired by corresponding conditions. On the $x$-axis is the proportion of participants in each scenario in Experiment 1 who were given the full reference point, conditional on whether they selected the empty frame or the full frame (or $p$ [container was full | speaker's frame]). On the $y$-axis is the proportion of participants in each condition in Experiment 3 who inferred that the speaker's reference point was full, conditional on the frame presented to them (or $p$ [listener infers container was full | speaker's frame]). The data points generally lie close to the identity line, showing that the listeners tended to infer a particular reference point, given a particular frame, about as often as that really was the speakers' reference point, given their selected frame. 
was the listener analogue to (part of) Experiment 2, the medical treatment experiment. The results are shown at the bottom of Table 1 (under "Experiment 4"). ${ }^{3}$ Just as the speakers in Experiment 2 were more likely to describe a new treatment in terms of \% die when the old treatment led to a higher (rather than lower) survival rate, the listeners in Experiment 4 were more likely to infer that the old treatment led to a higher survival rate $(100 \%$ rather than $0 \%$ ) when the new treatment outcome was framed as "50\% die" rather than "50\% survive" $\left[\chi^{2}(1, N=124)=\right.$ $3.99, p=.046]$. It appears that speakers select frames systematically and that listeners exploit this fact.

\section{GENERAL DISCUSSION}

We set out to test three hypotheses. The first was that reference points would reliably influence speakers' choices between logically equivalent frames. Confirmation of this hypothesis can be seen in Table 1 for Experiments 1 and 2, where the percentage of participants selecting a particular frame differs for each pair, showing that manipulating reference points affected which frame was selected. Furthermore, the second percentage in each pair is larger than the first, supporting the theoretical account depicted in Figure 1: A given label (and hence, frame) is more likely to be preferred if that label's percentage has increased, rather than decreased, relative to a reference point.

The second hypothesis was that listeners would make different inferences about a speaker's reference points, depending on the speaker's choice of frame. Confirmation of this hypothesis can be seen in Table 1 for Experiments 3 and 4 , where the percentage of participants inferring a particular reference point differs within each pair, showing that manipulating frames affected which reference point was inferred. Furthermore, the second percentage in each pair is larger than the first, also indicating that the theoretical account depicted in Figure 1 is correct.

The third hypothesis was that listeners' inferred reference points would match the actual reference points that influenced the speakers. Evidence for this hypothesis can be seen in Figure 2, which shows that the listeners in Experiment 3 tended to infer a particular reference point, given a particular frame, about as often as that really was the speakers' reference point in Experiment 1, given the speaker's chosen frame.

These results provide insight into why framing effects are so robust: Frames that are logically equivalent nonetheless convey different information regarding a speaker's reference point, and listeners are sensitive to this. The obvious benefit of this is that people can communicate efficiently. Describing a glass in terms of how empty (rather than how full) it is or describing a medical treatment in terms of its mortality (rather than survival) rate conveys information not just about its absolute status, but also about its relative status. ${ }^{4}$ (For an example of logically equivalent hypotheses being treated differently by speakers and listeners in an adaptive way, see McKenzie, Ferreira, Mikkelsen, McDermott, \& Skrable, 2001, and McKenzie \& Mikkelsen,
2000; for an example of logically equivalent observations in a covariation task being treated differently for normatively defensible reasons, see McKenzie \& Mikkelsen, in press.)

Although being sensitive to the implicit information contained in frames can facilitate communication, the downside is that listeners can be manipulated or misled. For instance, Thaler (1980) noted that credit card companies lobbied extensively to label the difference between a product's cash price and its credit price a "cash discount" (implying that the credit price is the reference point) rather than a "credit surcharge" (implying that the cash price is the reference point), presumably because people would be more likely to pass up a discount than incur a surcharge. Similarly, following Levin and Gaeth's (1988) results, advertisers might choose to describe ground beef in terms of $X \%$ lean regardless of its fat content, in order to evoke a more favorable impression (see also Sanford et al., 2002). The framing literature indicates that selecting frames with an eye toward manipulating behavior will often be successful. (Note that deceptive speakers can always defend their frame selection by pointing out that the alternate frame is logically equivalent.) Listeners generally appear to assume that speakers are sincerely communicating their reference point. When this assumption is violated-when experimenters randomly assign participants to different frame conditions in the laboratory or when advertisers intentionally select positive frames-listeners can be misled.

We mentioned that the notion that listeners infer reference points on the basis of frames is consistent with most authors' view of risky choice framing effects. However, Levin et al. (1998; Levin \& Gaeth, 1988) argue that attribute-labeling framing effects, such as the ground beef example, occur because a negative frame (e.g., percentage of fat) leads people to focus on the object's negative attributes, whereas a positive frame (e.g., percentage of lean) leads to focusing on positive attributes. Our data suggest that, as with risky choice framing effects, it is the implied reference point of the different frames that, at least in part, accounts for attribute framing effects. Describing beef in terms of how fat it is implies that it is relatively fat, whereas describing it in terms of how lean it is implies that it is relatively lean. Consistent with our account, Levin and Gaeth found that the percentage of fat frame resulted in higher subjective ratings of fat content than did the percentage of lean frame even before the product was consumed. From our perspective, this was a straightforward inference that participants made on the basis of the presented frame, not a result of biased processing of information.

A potential shortcoming of our experiments is that the participants were presented with forced-choice tasks, which might result in different behavior relative to more natural situations in which speakers spontaneously select frames and listeners spontaneously interpret them. Future research on this topic should include less constrained laboratory tasks or, perhaps, even an analysis of naturally occurring discourse. It should not be assumed, however, that 
less constrained situations will lead to weaker results than those reported here. Indeed, it is plausible that more natural settings will lead speakers to be even more reliably influenced by reference points and will lead listeners to be even more sensitive to the selected frame.

In sum, our data suggest that frames reliably communicate implicit information in addition to their literal content. Reference points influence the frame chosen by a speaker, and listeners tend to infer those same reference points. Thus, frame selection serves an efficient communicative purpose, assuming that speakers select frames depending on their reference point. In the context of everyday discourse, it is reasonable for listeners to make this assumption. However, when this crucial assumption is violated (e.g., as it is in the laboratory), listeners can be misled or manipulated.

\section{REFERENCES}

FischHoff, B. (1983). Predicting frames. Journal of Experimental Psychology: Learning, Memory, \& Cognition, 9, 103-116.

Frisch, D. (1993). Reasons for framing effects. Organizational Behavior \& Human Decision Processes, 54, 399-429.

Hilton, D. J. (1995). The social context of reasoning: Conversational inference and rational judgment. Psychological Bulletin, 118, 248-271.

Kahneman, D., \& Tversky, A. (1979). Prospect theory: An analysis of decision under risk. Econometrica, 47, 263-291.

Kahneman, D., \& Tversky, A. (1984). Choices, values, and frames. American Psychologist, 39, 341-350.

KüHBERGER, A. (1998). The influence of framing on risky decisions: A meta-analysis. OrganizationalBehavior \& Human Decision Processes, 75, 23-55.

LeVin, I. P., \& GAETh, G. J. (1988). How consumers are affected by the framing of attribute information before and after consuming the product. Journal of Consumer Research, 15, 374-378.

Levin, I. P., Schneider, S. L., \& Gaeth, G. J. (1998). All frames are not created equal: A typology and critical analysis of framing effects. OrganizationalBehavior \& Human Decision Processes, 76, 149-188.

LeVIn, I. P., SchnitTJer, S. K., \& Thee, S. L. (1988). Information framing effects in social and personal decisions. Journal of Experimental Social Psychology, 24, 520-529.

Marteau, T. M. (1989). Framing of information: Its influence upon decisions of doctors and patients. British Journal of Social Psychology, 28, 89-94.

McKenzie, C. R. M., Ferreira, V. S., Mikkelsen, L. A., McDermott, K. J., \& Skrable, R. P. (2001). Do conditional hypotheses target rare events? Organizational Behavior \& Human Decision Processes, 85 , 291-309.

McKenzie, C. R. M., \& Miknelsen, L. A. (2000). The psychological side of Hempel's paradox of confirmation. Psychonomic Bulletin \& Review, 7, 360-366.

McKenzie, C. R. M., \& Miknelsen, L. A. (in press). A Bayesian view of covariation assessment. Cognitive Psychology.

McNeil, B. J., Pauker, S. G., Sox, H. C., JR., \& Tversky, A. (1982). On the elicitation of preferences for alternative therapies. New England Journal of Medicine, 306, 1259-1262.

Meyerowitz, B. E., \& Chaiken, S. (1987). The effect of message framing on breast self-examination attitudes, intentions, and behavior. Journal of Personality \& Social Psychology, 52, 500-510.

Moxey, L. M., \& SAnford, A. J. (2000). Communicating quantities: A review of psycholinguistic evidence of how expressions determine perspectives. Applied Cognitive Psychology, 14, 237-255.

Sanford, A. J., Fay, N., Stewart, A., \& Moxey, L. (2002). Perspective in statements of quantity, with implications for consumer psychology. Psychological Science, 13, 130-134.

SCHNEIDER, S. L. (1992). Framing and conflict: Aspiration level contingency, the status quo, and current theories of risky choice. Journal of Experimental Psychology: Learning, Memory, \& Cognition, 18, 1040-1057.

SCHWARz, N. (1996). Cognition and communication: Judgmental biases, research methods, and the logic of conversation. Mahwah, NJ: Erlbaum.

THALER, R. (1980). Toward a positive theory of consumer choice. Journal of Economic Behavior \& Organization, 1, 9-60.

TVersky, A., \& Kahneman, D. (1981). The framing of decisions and the psychology of choice. Science, 211, 453-458.

Tversky, A., \& Kahneman, D. (1986). Rational choice and the framing of decisions. Journal of Business, 59, S251-S278.

Wilson, D. K., Kaplan, R. M., \& Schneiderman, L. J. (1987). Framing of decisions and selections of alternatives in health care. Social Behaviour, 2, 51-59.

\section{NOTES}

1. Fischhoff (1983) examined how participants conceptualized a decision problem involving a risky and a riskless option. After reading the problem, the participants were presented with "three ways one might think about this problem" (i.e., three different frames). They then selected the most (and least) "natural" frame, which is at least superficially related to some of our experiments. However, the frames presented to the participants did not differ in terms of the implied reference point, nor was the reference point implied by the decision problem itself manipulated. Unlike our experiments, Fischhoff's generally did not address the communicative aspect of framing and, specifically, did not address the influence of reference points on speakers' frame selection.

2. We are not saying that the listeners' responses would have been optimal if all the data points were to lie on the identity line. Optimality would lead the listeners to always select the most likely reference point, given a frame, so the listeners would infer a particular reference point either $0 \%$ or $100 \%$ of the time. Furthermore, the analysis assumes equal prior probabilities for the full and the empty reference points. These prior probabilities accurately describe the situation in Experiment 1 but may or may not reasonably describe more realistic situations.

3. Details of the experiment are available from the first author.

4. It should be noted that speakers' reference points reflect beliefs, perceptions, or expectations and do not necessarily reflect something inherent in the external world. Thus, two speakers might legitimately have different reference points and, hence, select different frames. This does not mean, however, that the speakers' reference points are arbitrary or that the listeners' inferences are pointless. Being influenced by a speaker's reference point could be beneficial when the speaker is more experienced than the listener with the topic in question (e.g., when a physician describes a new treatment's outcome to a patient) or when the listener has (or would have, given the same information) the same reference point as the speaker. Indeed, an interesting empirical question is whether people tend to have the same reference points. To the extent that people do share reference points, speakers will frame outcomes the same way as listeners would frame them for themselves, given the same information. When a listener does not yet have an established reference point, sensitivity to a speaker's frame selection might be especially adaptive if the speaker and the listener tend to have similar reference points.

(Manuscript received October 13, 2001; revision accepted for publication July 12, 2002.) 